Marco Künne*, Sebastian Hagemeier, Eireen Käkel, Hartmut Hillmer und Peter Lehmann

\title{
Investigation of measurement data of low-coherence interferometry at tilted surfaces in the 3D spatial frequency domain
}

\author{
Untersuchung von Weißlichtinterferometer-Messdaten geneigter Oberflächen im 3D \\ Ortsfrequenzbereich
}

DOI 10.1515/teme-2021-0051

Zusammenfassung: In diesem Beitrag werden das 3DÜbertragungsverhalten von Interferenzmikroskopen und sein Einfluss auf die Eigenschaften von Interferenzsignalen insbesondere an geneigten Oberflächen untersucht. Die aufgenommenen Interferenz-Bilderstapel werden in den 3D-Ortsfrequenzbereich transformiert. Dies ermöglicht eine umfassende Signalanalyse, die durch das in einer vorangegangenen Publikation eingeführte Double Foil Model unterstützt wird. Dieses Modell erlaubt die Interpretation der Signalspektren und des zugrunde liegenden Übertragungsverhaltens des Interferometers. Dabei werden die relevanten Ortsfrequenzanteile durch das Konzept der Ewald-Kugel bestimmt, die durch die numerische Apertur (NA) des Mikroskopobjektivs begrenzt wird. Die 3D Frequenzbereichsanalyse zeigt unmittelbar, dass die laterale Ausdehnung der Übertragungsfunktion von der axialen Ortsfrequenz abhängt. In diesem Beitrag werden Messergebnisse von mittels Nanoimprint-Lithographie hergestellten Messobjekten untersucht. Die Charakteristika der zugehörigen Signalspektren können für eine verbesserte Analyse der Einhüllenden und der Phasenlage der während des Tiefenscans gemessenen Signale genutzt werden. Dabei werden die Interferenzsignale zunächst mit einem schmalen Bandpassfilter gefiltert, um die relevanten Signalfrequenzen zu extrahieren. Obwohl sich dabei die Form und Breite der Einhüllenden ändern, wird die $\mathrm{Zu}$ -

\footnotetext{
*Korrespondenzautor: Marco Künne, Sebastian Hagemeier, Peter Lehmann, Lehrstuhl für Messtechnik, Universität Kassel, Wilhelmshöher Allee 71, 34121 Kassel, E-Mail:

marco.kuenne@uni-kassel.de

Eireen Käkel, Hartmut Hillmer, Institut für

Nanostrukturtechnologie und Analytik (INA), Universität Kassel, Heinrich-Plett-Str. 40, 34132 Kassel

Hartmut Hillmer, Peter Lehmann, Center for Interdisciplinary Nanostructure Science and Technology (CINSaT), Universität Kassel, Heinrich-Plett-Str. 40, 34132 Kassel
}

verlässigkeit der Topographiebestimmung verbessert. Dies gilt insbesondere für stark geneigte Oberflächenbereiche.

Schlüsselwörter: Kurzkohärente Interferometrie, 3D Ortsfrequenzraum, Signalfilterung, Oberflächenneigung.

Abstract: The 3D transfer characteristics of interference microscopes and their effect on the interference signals occurring at surface slopes are studied. The interference image stacks acquired during a depth scan are 3D Fourier transformed. This allows a comprehensive frequency domain analysis of the interferograms. The double foil model introduced in a previous publication enables the interpretation of the signal spectra and the underlying transfer behavior of the interferometer using the concept of the Ewald sphere, which is limited by the numerical aperture (NA) of the imaging system. Analysis in the 3D spatial frequency domain directly discloses that the lateral dimensions of the transfer function depend on the axial spatial frequency. In this contribution we investigate measuring objects produced by Nanoimprint-Lithography. The corresponding signal spectra bear information that can be utilized to optimize the subsequent signal processing algorithms. These include envelope and phase evaluation procedures of the interference signals. A narrow bandpass filter is used to actively select certain frequency components in order to improve the robustness of the estimation of the envelope position. Although the shape and width of the envelope are affected, this procedure increases the reliability of the evaluation process and improves the accuracy of the measured topography especially at steeper surface slopes.

Keywords: Coherence scanning interferometry, 3D spatial frequency domain, signal filtering, surface slope. 


\section{Introduction}

Coherence scanning interferometry (CSI) is one of the most common optical profiling methods [5, 13]. Due to the trend towards miniaturization in micro- and nanotechnology the lateral resolution capability of optical instruments is of increasing relevance. In order to improve the optical resolution, shorter illumination wavelengths can be used as well as objectives with higher numerical aperture (NA). The use of a higher NA in CSI leads to the well known NA-effect characterized by an increased fringe spacing of the interferograms which can be interpreted as an increased effective wavelength $\lambda_{\text {eff }}[4,16]$. In a previous study the spectral composition of interferograms obtained from measurements at different grating periods is analyzed [11]. The results demonstrate that different spectral components of an interference signal due to different corresponding incident and reflection angles, provide different lateral resolution. This contribution shows that the local tilt of a surface has a strong influence on the spectral composition of the signals. Here, we investigate how this affects the results of phase and envelope analysis in CSI measurements of nanofabricated surfaces. To gain deeper insight measurement data will also be analyzed and discussed with respect to their 3D spatial frequency representation.

If the full pupil plane of a microscope objective is illuminated incoherently, light hits the sample under different incident angles $\Theta_{\mathrm{i}}$, where each angle is attributed to a distinct wave vector $\vec{k}_{\mathrm{i}}$ of the light corresponding to a certain spatial frequency. The maximum incident angle $\Theta_{\max }=\arcsin (\mathrm{NA})$ defined by the NA of the objective is directly related to Abbe's resolution criterion for the smallest resolvable grating period $\delta=\lambda_{0} /\left(2 \sin \Theta_{\max }\right)=$ $\lambda_{0} /(2 \mathrm{NA})$, where $\lambda_{0}$ is the central wavelength of the light source. Additionally, the angle $\Theta_{\max }$ fundamentally limits the surface slopes that can be measured. Light reflected at a specular local surface area, whose slope exceeds $\Theta_{\max }$, will not reach the objective lens. Figure 1 shows the reflection at a tilted surface and the wave vectors collected by the objective lens. Only the incident and reflected wave vectors $\vec{k}_{\mathrm{i}}$ and $\vec{k}_{\mathrm{r}}$, which are inside the overlapping region of the two light cones, contribute to the interference signal. For this study we consider a Linnik-type interferometer consisting of two identical high NA objectives in the measurement and the reference arm.

If there is a perfectly aligned planar mirror in both arms, all rays at incident angles $\Theta_{\mathrm{i}}$ will be reflected towards the objective lens. During the CSI measurement process, a depth scan of the measurement object is per-

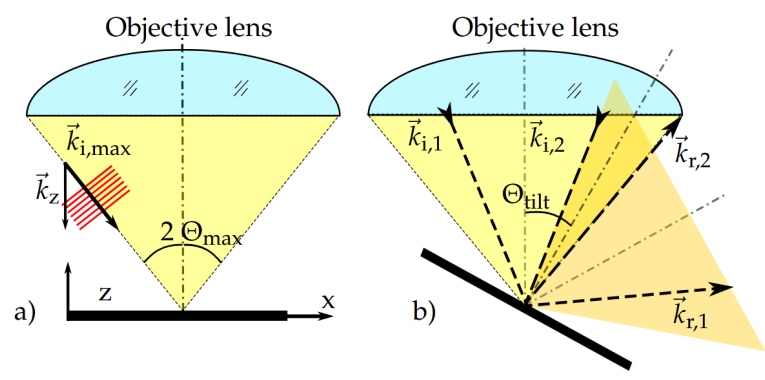

Fig. 1: Schematic of the reflection at a mirror perpendicular to the optical axis of the objective lens (a) and at a tilted mirror (b). The overlap of the two light cones in the right drawing determines, which wave vectors contribute to an interference signal.

formed. Here, the axial distance between interferometer and the measuring object is changed in equidistant steps, while interference patterns are recorded by a camera. In this way an interference signal occurs for each camera pixel. Since only the z-position of the sample changes during the process, only the z-components $k_{\mathrm{z}}$ of the wave vectors $\vec{k}$ contribute to the recorded change of the interference. For each wave vector reaching the sample under a different incident angle an effective wavelength $\lambda_{\text {eff }}$ can be calculated:

$$
k_{\mathrm{z}}=\vec{k}_{\mathrm{i}} \cdot \hat{z}=k_{0} \cos \left(\Theta_{\mathrm{i}}\right)=\frac{2 \pi}{\lambda_{0}} \cos \left(\Theta_{\mathrm{i}}\right)=\frac{2 \pi}{\lambda_{\text {eff }}},
$$

with $\hat{z}$ being the unit vector in z-direction. This means that the height distance between fringes in the interference pattern is not directly determined by the illumination wavelength $\lambda_{0}$ but by the effective wavelength $\lambda_{\text {eff }}$. This NA-effect [16] along with the angle selection at tilted surfaces plays an important role with respect to the shape of the interference signal and its underlying spectrum.

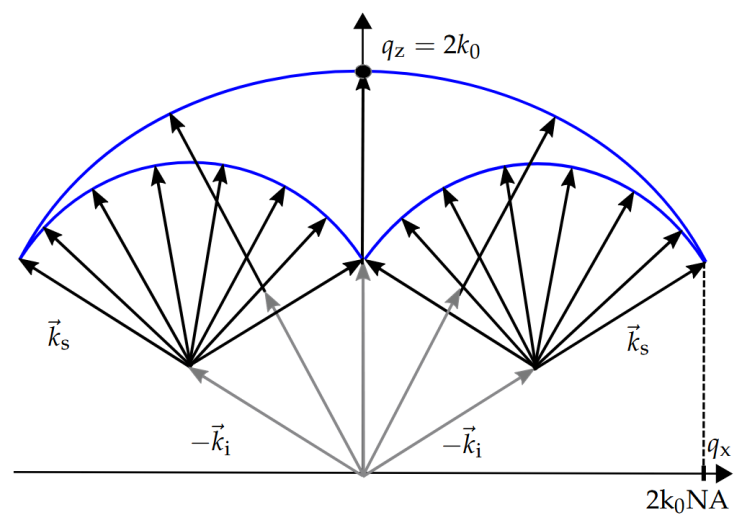

Fig. 2: Ewald sphere for a limited NA defined by all vectors $\vec{q}=$ $\vec{k}_{\mathrm{s}}-\vec{k}_{\mathrm{i}}$ contributing to the image formation with $\vec{k}_{\mathrm{s}}$ (black) and $\vec{k}_{\mathrm{i}}$ (grey) being the scattered and incident wave vectors. 
By 3D Fourier transforming the measured image stack, a frequency representation of the signal can be obtained in terms of the spatial frequency vector $\vec{q}=\vec{k}_{\mathrm{s}}-\vec{k}_{\mathrm{i}}[12]$. When considering all possible incident and scattering angles, this is analogous to the construction of the Ewald sphere as depicted in Fig. 2. Based on Eq. 1 for specular reflection the $\vec{q}$-vectors will have the simple form

$$
\vec{q}=\left(\begin{array}{c}
0 \\
0 \\
q_{\mathrm{z}}
\end{array}\right), \quad \text { with } \quad q_{\mathrm{z}}=2 k_{0} \cos \left(\Theta_{\mathrm{i}}\right)=\frac{4 \pi}{\lambda_{\text {eff }}} .
$$

However, if a tilted or even curved surface is considered the $q_{\mathrm{x}}$ and $q_{\mathrm{y}}$ components of the vector $\vec{q}$ will no longer be zero. If the local surface slope covers all angles up to $\Theta_{\max }$ the 3D frequency representation of the image stack will cover the whole Ewald sphere limited in $q_{\mathrm{x}}$ and $q_{\mathrm{y}}$ direction by twice the NA of the system and in $q_{\mathrm{z}}$ direction by the illumination wavelength $\lambda_{0}$. The maximum and minimum $q_{\mathrm{z}}$-values are defined by $q_{\mathrm{z}, \max }=2 k_{0}$ and $q_{\mathrm{z}, \min }=2 k_{0} \cos \left(\Theta_{\max }\right)$, respectively. This concept has been further developed by Sheppard et al. [15] for studying the transfer function of confocal microscopes. More recently this approach was used to investigate the transfer behaviour of scanning interferometers $[3,12,17]$. Spatial frequency analysis in the $\vec{q}$-space gives insight into the spectral composition of interference signals at steep surface slopes and provides support for subsequent signal processing.

\section{Signal processing}

In our signal processing algorithm the measured interference signal $I(z)$ for a single camera pixel is first bandpass filtered by multiplying the signal spectrum with a Gaussian in the frequency domain. Usually, the Gaussian is chosen in a way that its central wavenumber $k_{0, \text { filter }}$ aligns with the central wavenumber of the interference signal and no significant spectral component of the signal is suppressed. Figure 3 shows the absolute value of a signal spectrum before and after the bandpass filtering process for two different filter wavenumbers and widths. After filtering, the envelope is computed via Hilbert transform $[2,18]$. By calculating the maximum position of the envelope a corresponding height value can be obtained and thus the topography can be reconstructed. Additionally, the phase of the signal can be used to determine a height value. This method is more precise on a small scale, but suffers from $2 \pi$-ambiguity because for the calculation of the phase $\varphi$ at a single camera pixel at the position $x_{0}, y_{0}$ the arctan function is used:

$$
\varphi\left(k_{\mathrm{z}, \mathrm{eval}}, x_{0}, y_{0}\right)=\arctan \left(\frac{\Im\left\{S\left(k_{\mathrm{z}, \mathrm{eval}}\right)\right\}}{\Re\left\{S\left(k_{\mathrm{z}, \mathrm{eval}}\right)\right\}}\right) .
$$

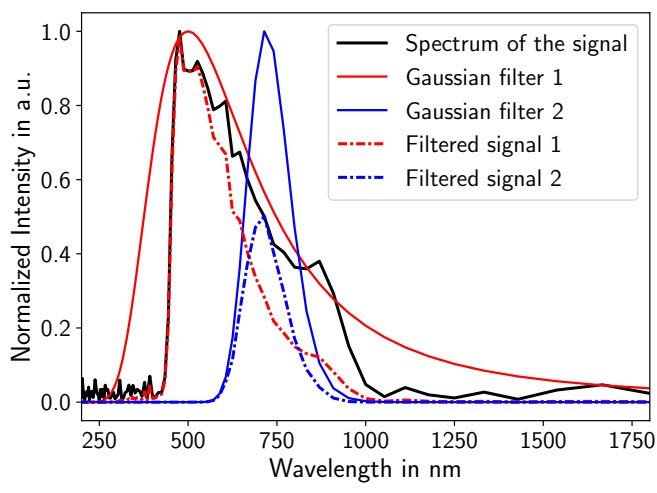

Fig. 3: Absolute values of the spectrum of an interference signal, two different filter functions centered at $\lambda_{0, \text { filter }}=720 \mathrm{~nm}$ with a FWHM of $138 \mathrm{~nm}$ (blue) and at $\lambda_{0, \text { filter }}=500 \mathrm{~nm}$ with a FWHM of $374 \mathrm{~nm}$ (red) and the corresponding filtered signal spectra.

Here, $S\left(k_{\mathrm{z}, \text { eval }}\right)$ is a complex single frequency Fouriercoefficient of the signal at the frequency $k_{\mathrm{z} \text {,eval }}=2 \pi / \lambda_{\text {eval }}$, where $\lambda_{\text {eval }}$ is usually chosen to match $\lambda_{0, \text { filter }}$ so that $k_{\mathrm{z}, 0}=k_{\mathrm{z}, \mathrm{eval}}$. This coefficient is computed by a single frequency discrete Fourier transformation (DFT), also called Lock-In-Method [6, 9]. The frequency corresponds to the evaluation wavelength $\lambda_{\text {eval }}$, which is shown in this and previous studies $[10,11]$ to have a significant impact on the resulting topography.

Finally, the problem of $2 \pi$-ambiguity is solved by using the height information obtained from the envelope position to estimate the fringe order for the phase evaluation. For the remaining phase jumps a phase unwrapping algorithm can be used to obtain a continuous surface profile. For further information on the evaluation process and algorithm it is referred to [18].

\section{Results}

As an object of practical relevance hollow spherical structures etched in silicon (cf. Fig. 4a) are measured. The samples have been produced at the Institute for Nanostructure Technology and Analytics (INA) of the University of Kassel. The stuctures have been fabricated using deposition, lithograpy and etching and are used as molds in Nanoimprint Lithography and replicated several times [1]. They allow to study different slopes in a single measurement because of their curvature. Our Linnik-interferometer is equipped with two identical microscope objectives of $\mathrm{NA}=0.9$ and 100x magnification. The light source is a royal blue LED with a central wavelength of $\lambda_{0}=460 \mathrm{~nm}$ 

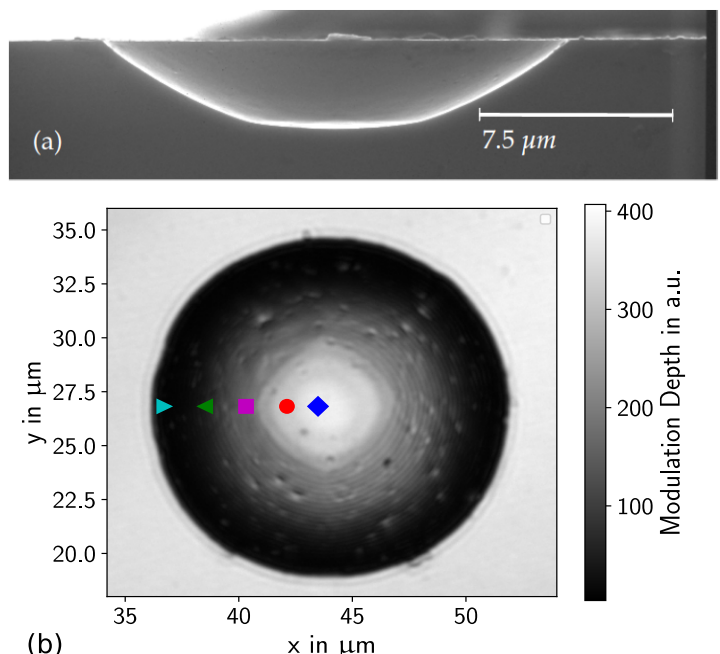

(b)

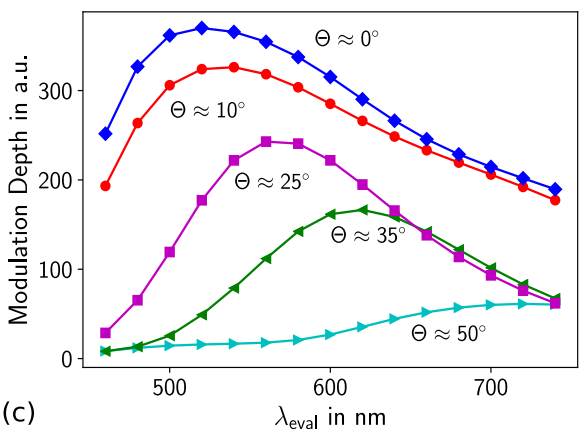

Fig. 4: (a) SEM picture of a profile of the measured structure, (b) modulation depth for $\lambda_{\text {eval }}=560 \mathrm{~nm}$ of a concave structure. (c) shows the modulation depths for the locations indicated by the markers in (b) for different evaluation wavelengths $\lambda_{\text {eval }}$.

and the image stacks are recorded with an axial step size $\Delta z$ of $20 \mathrm{~nm}$ by a scientific CMOS-camera. The modulation depth of interference signals obtained from a concave structure under investigation is shown in Fig. 4b. At the edge of the structure the modulation depth drops significantly, because at these points the slope of the structure is maximum and most of the light is lost (cf. Fig. 1). Close to the edge the tilt angle of the surface is approximately $50^{\circ}$, which according to Eq. 1 translates to an effective wavelength of $\lambda_{\text {eff }} \approx 720 \mathrm{~nm}$. An increase of $\lambda_{\text {eval }}$ approaching this wavelength would therefore lead to an increase of the modulation depth at the edge of the structure, while at the center the modulation depth decreases. For points at different slopes of the structure, which are marked in Fig. 4b, the modulation depth is plotted in Fig. 4c for a variety of evaluation wavelengths $\lambda_{\text {eval }}$. Throughout this study two different filter bandwidths are used. A broad bandpass filter (BBF), which covers almost all frequency components of the signal spectrum, and a narrow bandpass filter (NBF), which includes only a part of the signal
Table 1: Bandwidths of the Gaussian filters in the frequency domain and the corresponding FWHM in spatial frequency and wavelength units for three central wavelengths $\lambda_{0, \text { filter }}$.

\begin{tabular}{l|c|c|c|c|}
\hline Filter & $\Delta q_{z}$ (FWHM) & \multicolumn{3}{|c|}{$\Delta \lambda($ FWHM $)$} \\
\hline$\lambda_{0, \text { filter }}$ & - & $460 \mathrm{~nm}$ & $575 \mathrm{~nm}$ & $720 \mathrm{~nm}$ \\
\hline BBF & $16.65 \mu \mathrm{m}^{-1}$ & $310 \mathrm{~nm}$ & $513 \mathrm{~nm}$ & $889 \mathrm{~nm}$ \\
NBF & $3.33 \mu \mathrm{m}^{-1}$ & $56 \mathrm{~nm}$ & $88 \mathrm{~nm}$ & $138 \mathrm{~nm}$ \\
\hline \hline
\end{tabular}

spectrum. The central wavelength of the filter $\lambda_{0, \text { filter }}$ is always chosen to match the evaluation wavelength $\lambda_{\text {eval }}$. Table 1 shows the full width at half maximum (FWHM) of these filters for different central wavelength. Note that for the results shown in Fig. 4 the NBF is used. In Fig. 5 the corresponding profiles of the topography calculated from envelope and from phase are depicted. A commercial scanning confocal microscope (SCM) made by Nanofocus AG $[7,8]$ with $50 \mathrm{x}, \mathrm{NA}=0.95$ is used for comparison. It turns out that due to this filtering the result of envelope evaluation becomes more robust, if the central wavelength $\lambda_{0, \text { filter }}$ increases. This is due to the better noise suppression. The effect is more significant at steeper surface areas, because the determination of the envelope maximum becomes increasingly noise-susceptible if the signal gets weaker [14]. The maxima of the curves in Fig. 4c shift to higher effective wavelengths for points closer to
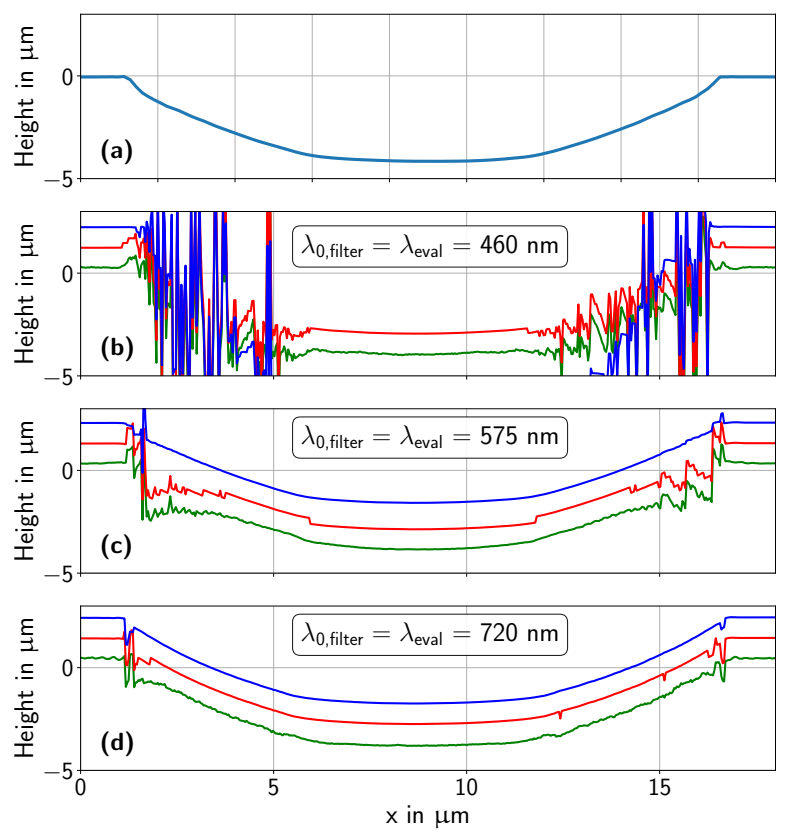

Fig. 5: Height profiles of the concave structure. (a) reference measurement obtained by a SCM, (b)-(d) results of CSI obtained from the signal evaluation of the envelope (green), phase (red) and the unwrapped phase (blue). In all cases the NBF with a central wavelength $\lambda_{0, \text { filter }}=\lambda_{\text {eval }}$ is used. 

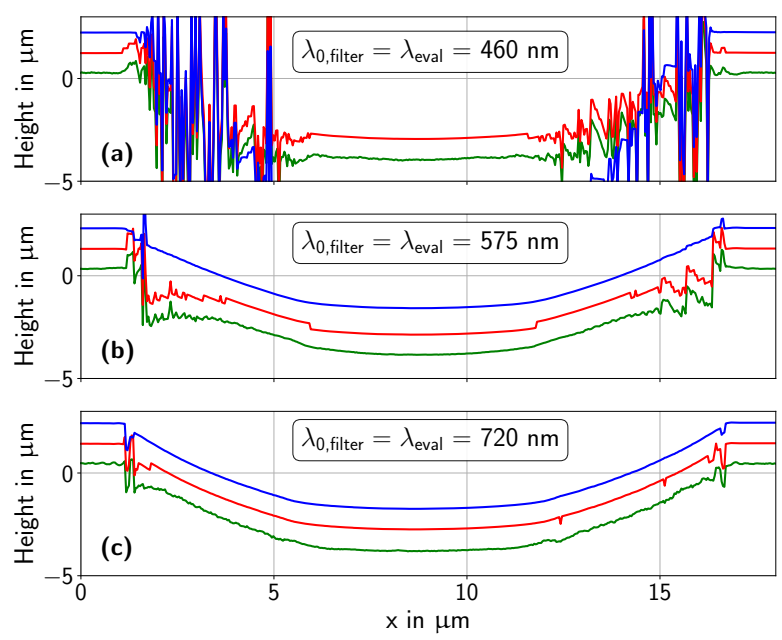

Fig. 6: Height profiles of the concave structure resulting from the envelope (green), phase (red) and unwrapped phase (blue). The BBF is used for the envelope calculation.

the edge of the structure. This is of course directly related to Eq. 1. The data corresponding to the point closest to the edge of the structure show a maximum at $720 \mathrm{~nm}$, which corresponds to the slope angle of $50^{\circ}$ at the edge. Evaluating a structure like this with a NBF, using the illumination wavelength $\lambda_{0}=460 \mathrm{~nm}$ for evaluation will not lead to reliable results for the surface topography, because the modulation depth is almost zero for points at steeper slopes (see Fig. 5b). Taking the increased fringe spacing due to the NA-effect into account $\lambda_{0}$ is multiplied by a factor of 1.25 , which is chosen based on the results of Sheppard et al. [16] to obtain an evaluation wavelength of $\lambda_{\text {eval }}=575 \mathrm{~nm}$. The results (see Fig. 5c) are reliable for small surface slopes, but strong deviations from the expected geometry occur at higher slopes, since for this wavelength the modulation depth is still not high enough. A third evaluation wavelength is chosen to be $\lambda_{\text {eval }}=720 \mathrm{~nm}$ (see Fig. 5b), based on the results of Fig. 4c. At this wavelength the modulation depth is sufficiently high for all points of the structure. The resulting surface profiles show jumps only at the edges of the structure. According to Fig. 4c, the modulation depth should still be sufficient for a reliable evaluation, but at the edges diffraction effects might disturb the signals. By unwrapping the phase evaluation results, smoother profiles can be obtained for $\lambda_{\text {eval }}=575 \mathrm{~nm}$ and $\lambda_{\text {eval }}=720 \mathrm{~nm}$. For $\lambda_{\text {eval }}=460 \mathrm{~nm}$ the phase unwrapping process does no longer work, since there are too many phase jumps occurring. Comparing these results to profiles obtained from the same signals but using the BBF gives insight into the effect of the filter width on the reconstructed topography. Figure 6 shows that a broad bandwidth filter leads to a
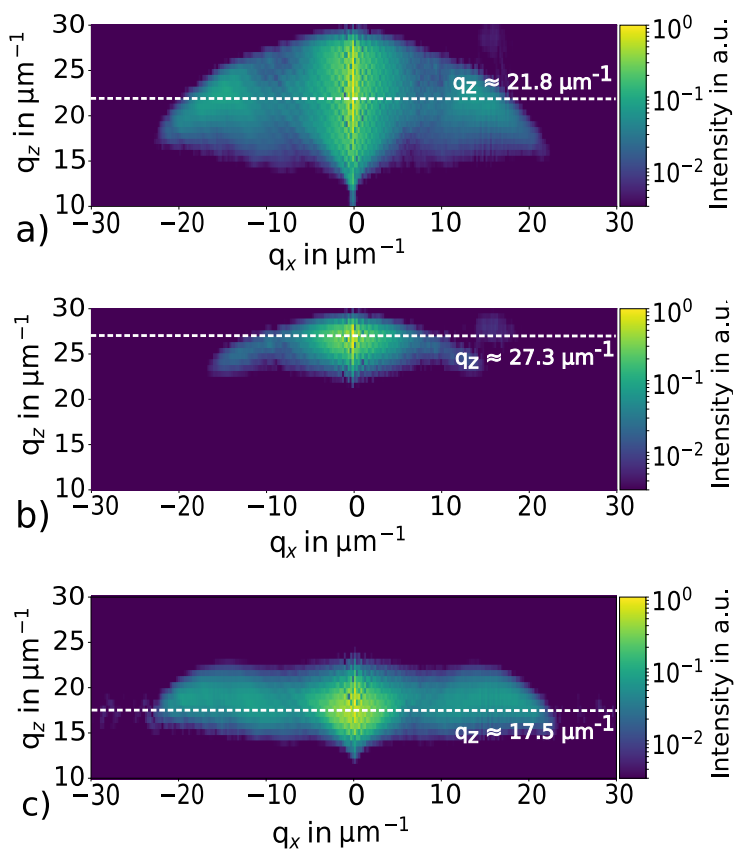

Fig. 7: Cross sections of the normalized intensity of the measurement data in the spatial frequency domain, using (a) the BBF at $\lambda_{0, \text { filter }}=575 \mathrm{~nm}$, (b) the NBF at $\lambda_{0 \text {, filter }}=460 \mathrm{~nm}$ and (c) the NBF at $\lambda_{0, \text { filter }}=720 \mathrm{~nm}$. The dashed horizontal lines correspond to the wavelength $\lambda_{\text {eval }}=\lambda_{0 \text {,filter }}$.

smooth topography for envelope and phase evaluation at small slopes, but fluctuations occur for steeper slopes even for higher central filter and evaluation wavelengths. Phase unwrapping decreases the number of height jumps and generates smoother profiles, but still phase jumps remain even at high evaluation wavelengths. The unreliable height values at the tilted surface sections in the results of envelope evaluation are caused by the previously discussed noise-susceptibility and can not be fully avoided when using a broad bandwidth filter. Looking at the spatial frequency representation of the filtered measurement data (Fig. 7) one can directly see the influence of the filter width and central wavelength on the data. The BBF at $\lambda_{0, \text { filter }}=575 \mathrm{~nm}$ covers all frequency contributions of the signal and only excludes high frequency noise (cf. Fig. 3). A NBF at $\lambda_{0, \text { filter }}=460 \mathrm{~nm}$ only includes the spatial frequency information for $q_{x}$ values up to $\approx \pm 12 \mathrm{\mu m}^{-1}$, which translates to a maximum slope of $26^{\circ}$. When filtering at $\lambda_{0, \text { filter }}=720 \mathrm{~nm} q_{x}$ values up to $22 \mu^{-1}$ are included, which correspond to a slope angle of $54^{\circ}$. For a more exact analysis a proper value for the out of plane angle $\phi_{\mathrm{S}}$ has to be taken into account, but nevertheless these results fit to the data presented in Figs. 4 and 5. For $q_{\mathrm{x}}$ values between $\pm 7 \mathrm{\mu m}^{-1}$ and $\pm 10 \mu^{-1}$ a decrease in intensity can be observed. The profiles in Figs. 5 and 6 
disclose that the slope is not increasing linearly. Between $x$ values of $6 \mu \mathrm{m}$ and $12 \mu \mathrm{m}$ there is a relatively flat area, which can be assigned to $\left|q_{x}\right|$ values of $<7 \mu \mathrm{m}^{-1}$, while outside this range the slopes become steeper. This unsteady rise in slope corresponds to the increased spectral intensity values at $\left|q_{x}\right|>10 \mathrm{~m}^{-1}$. For clarity, the intensity threshold in Fig. 7 is set to $2 \times 10^{-3}$ with respect to the maximum of the normalized intensity in order to eliminate background noise that is 3 orders of magnitude smaller than the maximum intensity.

\section{Conclusion}

The comparison of surface profiles shows that the reliability of the envelope evaluation at steep surface slopes can be significantly increased by filtering the signal with a NBF, even if spectral components of the signal get lost during this process. If a NBF is used, the position of

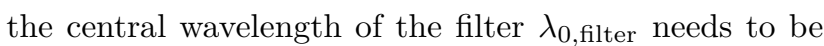
chosen in a way that the modulation depth is sufficient at all points of the sample, otherwise the topography can not be reconstructed correctly. Also $\lambda_{\text {eval }}$ needs to be adjusted along with the filter wavelength in order to perform a reliable phase evaluation. The best results for the measured structure could be obtained by using a narrow filter, combined with an evaluation wavelength of $\lambda_{\text {eval }}=\lambda_{0, \text { filter }}=720 \mathrm{~nm}$ matched to the effective wavelength $\lambda_{\text {eff }}$ at the maximum slope. These filter settings are quite unusual in CSI signal processing. If phase unwrapping is used in addition, a topography with almost no height jumps appears, which agrees quite well with the topography obtained by confocal microscopy.

Acknowledgment: The financial support of the Deutsche Forschungsgemeinschaft (DFG, LE 992/13-1, HI 763/16-1) is gratefully acknowledged.

\section{References}

[1] B. Allendorf, E. Käkel, U.-M. Ha, S. Hagemeier, H. Hillmer und P. Lehmann. Adaptive high-resolution linnik interferometry for $3 \mathrm{~d}$ measurement of microparticles. Optics Letters, 44 (14):3550-3553, 2019. 10.1364/OL.44.003550.

[2] S. S. Chim und G. S. Kino. Three-dimensional image realization in interference microscopy. Applied Optics, 31(14): 2550-2553, 1992. ISSN 1559-128X. 10.1364/AO.31.002550.

[3] J. Coupland und N. Nikolaev. Surface scattering and the 3d transfer characteristics of optical profilers. In Proc. of SPIE Vol 11352 (2020), S. 22. 10.1117/12.2556878.
[4] K. Creath. Calibration of numerical aperture effects in interferometric microscope objectives. Applied Optics, 28(16): 3333-3338, 1989. ISSN 1559-128X. 10.1364/AO.28.003333.

[5] P. de Groot. Principles of interference microscopy for the measurement of surface topography. Advances in Optics and Photonics, 7(1):1, 2015. 10.1364/AOP.7.000001.

[6] M. Fleischer, R. Windecker und H. J. Tiziani. Fast algorithms for data reduction in modern optical three-dimensional profile measurement systems with $\mathrm{mmx}$ technology. Applied Optics, 39(8):1290-1297, 2000. ISSN 1559-128X. 10.1364/ao.39.001290.

[7] S. Hagemeier und P. Lehmann. High resolution topography sensors in a multisenor measuring setup. In Proc. of SPIE Vol 11056 (2019). 10.1117/12.2525539.

[8] S. Hagemeier, M. Schake und P. Lehmann. Sensor characterization by comparative measurements using a multi-sensor measuring system. Journal of Sensors and Sensor Systems, 8 (1):111-121, 2019. 10.5194/jsss-8-111-2019.

[9] P. Lehmann, S. Tereschenko und W. Xie. Fundamental aspects of resolution and precision in vertical scanning whitelight interferometry. Surface Topography: Metrology and Properties, 4(2):024004, 2016.

[10] P. Lehmann, W. Xie, B. Allendorf und S. Tereschenko. Coherence scanning and phase imaging optical interference microscopy at the lateral resolution limit. Optics Express, 26 (6):7376-7389, 2018. 10.1364/OE.26.007376.

[11] P. Lehmann, S. Tereschenko, B. Allendorf, S. Hagemeier und L. Hüser. Spectral composition of low-coherence interferograms at high numerical apertures. Journal of the European Optical Society-Rapid Publications, 15(1):3775, 2019. 10.1186/s41476-019-0101-8.

[12] P. Lehmann, M. Künne und T. Pahl. Analysis of interference microscopy in the spatial frequency domain. Journal of Physics: Photonics, 3(1):014006, 2021. 10.1088/2515$7647 /$ abda15.

[13] D. Malacara. Optical Shop Testing. John Wiley \& Sons, Inc, Hoboken, NJ, USA, 2007. ISBN 9780470135976. 10.1002/9780470135976.

[14] J. Seewig, T. Böttner und D. Broschart. Uncertainty of height information in coherence scanning interferometry. In Proc. of SPIE Vol 8082 (2011). 10.1117/12.889796.

[15] C. Sheppard, T. J. Connolly und M. Gu. Imaging and reconstruction for rough surface scattering in the kirchhoff approximation by confocal microscopy. Journal of Modern Optics, 40(12):2407-2421, 1993. ISSN 0950-0340. $10.1080 / 09500349314552431$.

[16] C. Sheppard, K. G. Larkin und C. J. Sheppard. Effect of numerical aperture on interference fringe spacing. $A p$ plied Optics, 34(22):4731-4734, 1995. ISSN 1559-128X. 10.1364/AO.34.004731.

[17] R. Su, M. Thomas, M. Liu, J. Drs, Y. Bellouard, C. Pruss, J. Coupland und R. Leach. Lens aberration compensation in interference microscopy. Optics and Lasers in Engineering, 128:106015, 2020. ISSN 01438166. 10.1016/j.optlaseng.2020.106015.

[18] S. Tereschenko. Digitale Analyse periodischer und transienter Messsignale anhand von Beispielen aus der optischen Präzisionsmesstechnik. Dissertation, University of Kassel, Kassel, 2018. 